\title{
Simplicity, symmetry, and syntely: Stimulus measures of binary pattern structure
}

\author{
JOSEPH PSOTKA \\ Yale University. New Haven, Connecticut 06520
}

\begin{abstract}
A simple parameter free algorithm based solely on the sequential run and alternation structure of binary sequences is elaborated. The algorithm is designed to measure the sequence's syntely: the degree to which past consequences within a sequence converge on the continuation of that sequence's terminal run. This syntely algorithm is shown to predict subjects' expectancies in a sequential prediction task using short binary sequences. Other algorithms measuring the symmetry and simplicity of short hinary patterns are demonstrated, and their measures shown to be correlated with each other, but not correlated with syntely. Goodness and strength of expectancy are unrelated. The syntely algorithm is shown to be successful in predicting the error profiles of subjects learning short recurrent patterns of binary sequences. The syntely algorithm is based on the straightforward principle of induction by enumeration: the composite of past events controls the expectancy of future events. The success of this algorithm, even though it may embody the principle of induction only imperfectly, provides good evidence that this principle is a useful normative guide for understanding the human processing of contingencies in binary sequences, making complicated schemes of rules and hypotheses unnecessary.
\end{abstract}

An intelligent observer is quite able to use many different aspects of the information available in a stimulus. To begin the analysis of such information at a synoptic level, a simple dichotomy may usefully be created to distinguish two kinds of information by identifying information with structure and illustrating two sorts of structure, intrinsic and extrinsic (Garner, 1974). Extrinsic structure occurs when the stimulus denotes or signifies something other than itself, like the meaning contained in the word "dog," or the arithmetic progression inherent in the sequence of numbers (1 $3579 \ldots$ ). This sort of structure is beyond the scope of this paper. Instead, the subjective use of intrinsic structure as given by perceptible spatiotemporal regularities of the stimulus, like the optical and spatial frequency properties of "dog" or the single alternation structure of $(0 \times O X 0 X \ldots)$, will be analyzed at a very general level.

Information theory offers at its core the strikingly simplifying notion that information or structure can be discussed completely generally as it is embodied in the form of a binary time series like (XOXXOX...). The actual elements used in the series are completely irrelevant unless they designate some external structure. Since this is a real consideration with most, if not all, available elements, special precautions must be used in any practical case. Usually the simplest procedure to eliminate the effects of extrinsic structure on the results obtained with one sequence like the example above is to make sure that the same results are also obtained with its

I am grateful to W. R. Gamer and Michael Kubovy for their comments on an earlier draft. This research was supported by Grant MH 14229 from the National Institute of Mental Health to $Y$ ale University. The author is now at the University of Waterloo, Waterloo, Canada N2L 3G1. complementary sequence, in this case $(0 \times 00 \times 0 \ldots)$. The results are then dependent on the intrinsic structure of the sequence, and the elements themselves can be considered to be intrinsically neutral.

\section{MEMORY AND PROBABILITY LEARNING}

There already exists a large and established body of work on one aspect of the intrinsic structure of binary time series. This is the literature on partial reinforcement conditioning and probability leaming (Jenkins \& Stanley, 1950). More recent results in this tradition are summarized by Jones (1971). One of the clearest results of probability learning experiments with humans on binary patterns was the finding that the mean results across subjects and blocks of trials indicated that event predictions occurred in the same proportion as events in the sequence: probability matching occurred. Although this result presented some difficulty for an explanation based on a simple minded decision theory that predicted subjects should predict the more frequent alternative consistently on every trial, it quickly became apparent that subjects were responding to patterns of events, and the stimulus for responding on each trial was a composite of whatever experiences they had on previous trials (Hake \& Hyman, 1953; Nies, 1962).

An explanation of probability matching in terms of the ability of subjects to use subsequences of a series as cues to the future behavior of the series presupposed that they were able to remember at least short repeating sequences of binary events. That humans have this ability has been shown in many experiments for sequences of single alternation (Anderson, 1960), double alternation (Schoonard \& Restle, 1961), and short sequences like (X X O) (Goodnow \& Pettigrew, 1956) or 
(O O X O X) (Galanter \& Smith, 1958), or many other sequences (Garner \& Gottwald, 1967; Restle, 1967; Royer \& Garner, 1966) at varying rates, starting points, and modalities of presentation (Garner \& Gottwald, 1968; Handel \& Buffardi, 1968; Preusser, Garner, \& Gottwald, 1970), and with a variety of tasks to test memory (Alexander \& Carey, 1968; Glanzer \& Clark, 1962). In an experiment on probability learning using a complicated Markov sequence, Feldman and Hanna (1966) were able to show that event predictions matched the conditional probabilities following event states up to a length of five events at least.

In spite of this very clear evidence that subjects are quite capable of remembering event sequences of all sorts of patterns, attempts at making use of this information in order to understand the patterns of expectancy in probability learning experiments have used only a tiny proportion of it: memory for runs of identical events. The clearest expression of a model to describe subjects' expectancy based on memory for runs was given by Restle (1961). This model had only limited success (Restle, 1966). Modified versions of Restle's runs model have fared little better (Gambino \& Myers, 1967). It seems necessary to take into account the basic fact that subjects are able to remember and respond to patterns other than runs (Butler, Myers, \& Myers, 1969; Jones, 1971) if an adequate description of choice behavior in a binary prediction situation is to be achieved.

\section{INDUCTION AND EXPECTANCY}

Expectancy in a binary prediction paradigm may profitably be viewed as the result of inductive inference. The principle of induction may be stated simply in a form that is very similar to Newton's first law of motion. An induction is made by assuming that the present state of nature will continue into the future, and any consequences of events in the past will follow the same events in the future. Induction is to be distinguished from cross induction (Reichenbach, 1968) or analogy where the consequences of one set of events are predicted from another set of similar or different events. An example of a cross induction would be to predict the continuation of ( $0 \times X 000 \times X X X \ldots ?)$ on the basis of the learned arithmetic sequence ( $12324 \ldots$ ?). The distinction between induction and cross induction is related to the distinction between intrinsic and extrinsic structure. Inductions are based on the intrinsic structure of events, whereas cross inductions are based on their extrinsic structure. Since this paper is only concerned with intrinsic structure, it makes use only of the principle of induction. This basic principle that underlies all scientific achievement finds a ready application in the analysis of subjects' expectancy when predicting binary series.

Consider a short sequence of eight equally likely binary events like $(0 \times X \times 0 O X 0)$ as it is read from left to right. What inference may be drawn from this sequence about what will happen next (OXXXOOXO...?)? Using the basic principle of induction, events the same as the right-hand or terminal portion of the sequence may be sought in the left-hand or preceding portion of the sequence, their following events or consequences noted, and the inference drawn that these same consequences will next occur. This is induction by enumeration (Reichenbach, 1968). The frequencies of events following the terminal state in the past are evaluated and expected to occur with the same frequencies in the future.

This principle of induction is so simple and clear, it would seem to be nicely suited to determining the strength of expectancies by numerical calculation. However, it should be clear from the outset that this calculation cannot be performed on the basis of ideal equations. The necessary matching of the terminal state to all previous states within a sequence of finite and, in fact, relatively short length guarantees that no general mathematical solution is possible and an algorithm, a step by step calculation, is necessary. Also, an algorithm that tries to put into practice the theoretical principle of induction has to take into account practical details in order to deal with the performance of real people that theory may easily discount, like the accuracy of memory for past events. These are practical problems that must eventually be subjected to experimental investigation themselves, but in the beginning they can only be handled through careful experimental control and by drawing certain assumptions. The assumptions necessary for applying the principle of induction to short binary sequences will be considered next, after a definition of the terms used to describe the structure of binary patterns.

\section{DEFINITIONS}

In order to describe the intrinsic structure of binary patterns and the operation of algorithms designed to measure this structure, a number of terms will be used repeatedly in the ensuing discussion. Some of these words have been used in several different ways before in the sequential processing literature. It would seem advisable to provide definitions of these particular terms as they are used in this paper.

\section{Alternation}

An alternation occurs when an element is followed by the complementary element to its right in the binary sequence (for example, either $\mathrm{XO}$ or $\mathrm{OX}$ ).

\section{Run}

A run occurs when an element is followed by the identical element to its right in the binary sequence (for example, either $\mathrm{XX}$ or $\mathrm{OO}$ ). 


\section{Terminal Run}

A string of adjacent identical elements at the rightmost extreme of the binary sequence preceded by the complementary element constitutes the terminal run of the sequence (for example, the string of four Xs in XOXOXXXX).

\section{Matching Subsequences}

Subsequences within a pattern can be said to match if they form the identical sequence of runs and alternations. Thus, within (XXOXOOXO) there are a very large number of subsequences of various lengths that are matched at least once within the pattern. As an illustration, the longest subsequences that match within this pattern are XXOX and OOXO, each composed of four elements in length. But each of these subsequences may be further broken down into two subsequences of Length 3 that match, three subsequences of Length 2 that match, and the degenerate case containing no runs or alternations of four subsequences of Length 1 that match.

\section{ASSUMPTIONS OF THE SYNTELY ALGORITHM}

The cognitively salient features of a binary sequence are the runs and alternations (Butler, Myers, \& Myers, 1969; Jones, 1971; Royer \& Garner, 1966). It is assumed that the induction is based on the pattern of runs and alternations, so that the prediction is one of continuing a run or an alternation, and the states in the past that are evaluated are subsequences of runs and alternations.

It is assumed that terminal runs are unitized in a gestalt sense on the basis of identity and proximity. It is assumed that as a unit no inductions about the future can be drawn from itself, unless nothing else is available. Thus, the continuation of (XOXOXXXX) is based on (XOXO ....) and not on (... XXXX).

Using this simple principle of induction, the pattern of a sequence provides the intrinsic structure from which the alternatives governing expectancy are chosen. The expected continuation of a pattern may thus be derived from a straightforward analysis of the pattern itself. It is important to observe, however, that this analysis does not take into account any asymmetry in the frequency of the elements, since it deals only with the pattern of runs and alternations. Since it is clear that relative frequencies of events have a strong effect on expectancy, it remains for fyture analysis to show how relative frequencies and conditional relative frequencies interact.

The remainder of this paper provides a detailed system for the analysis of the intrinsic structure of binary patterns as this structure affects expectancy. This analytic system is given in the form of a syntely algorithm, where syntely (from the Greek sun, same or together, plus telos, completion or end) is the degree to
Which past conseguences converge on the continuation of the terminal run, expressed as a number ranging from 0 10 1. This neolugism, syntely, has been coined to provide a word whose meaning relates to the stimulus in a similar way that the strength of expectancy relates to the organism. Thus. syntely, like symmetry, describes something about the stimulus. The similarity of this analytic system to a symmetry algorithm is then exposed, and their relation to the simplicity of the pattern explored. Experimental evidence corroborating this analysis is given.

\section{SYNTELY: THE STRENGTH OF STIMULUS CONTINUATION}

An algorithm is like a game: It is easy to show someone how to play, but a formal description of the rules is often unwieldy and cumbersome. Here are the rules for calculating the syntely of any short binary pattern.

1. Record the length of the terminal run. For example, in (a) (XOXXXOOOO), the terminal run, underlined, is three elements long. In (b) $(\mathrm{O} \times \mathrm{OXOXOX})$ the terminal run, underlined, is only one element long.

2. Compare all of the rightmost or terminal segment of the original sequence with the subsequences immediately to the left by shifting a duplicate sequence underneath and to the left by the number of elements in the terminal run:
(a) $\times 0 \times X \times 000$
(b) $\mathrm{OXOXOXOX}$
$\mathrm{XOX \times X000}$
OXOXOXOX

3. The subsequences may now be matched vertically beginning with the rightmost element in the lower row. Since expectancy is assumed to depend on the pattern of runs and alternations in the sequence (not on the elements of the sequence themselves), match the rightmost, first element in the duplicate automatically and then look only to see if the pattern to the left in the duplicate is the same as in the to-be-matched subsequences in the original. This is true of both examples, since in a the second element from the right in the lower row duplicate forms part of a run with the rightmost element, as do the two elements directly above them, and in $b$ the same two pairs form alternations. If matches are found, as in these examples, the vertical comparison continues to the left; otherwise, it stops. In the examples Sequence b matches itself perfectly after the first shift to the left (all seven vertical comparisons match). Sequence a matches only the first four elements from the right.

4. The number of different elements matched in this fashion are, then, the number of matched subsequences in the sequence that have the same consequent and match the terminal segment. The consequent, either a 
run or an alternation, is determined by whether the element to the right of the matched subsequences in the upper row continues a run or an alternation with those matched subsequences. In the two examples these consequents are both alternations, as indicated below with matched subsequences underlined and matches indicated by ms:

(a) $\mathrm{XOXXX}-000$

$\mathrm{XOXXX000}$

$\mathrm{m} \mathrm{m} \mathrm{m} \mathrm{m} \cdot$ alternation

(b) $\frac{O X O X O X O}{X O X 0 X 0 X}-X$

$\mathrm{mm} \mathrm{m} \mathrm{m} \mathrm{m} \mathrm{m} \mathrm{-} \mathrm{alternation}$

5. The duplicate sequence is then shifted left by one element and the same procedure of matching is followed again. However, if the number of matched elements is less than the length of the terminal run and less than the number of matched elements on any previous line, it is not recorded. In the two examples this shift results in:

(a) $\mathrm{XOXXXOO}$

$\mathrm{X0X \times X000}$

(mm-run)

(b) $0 \times 0 \times 0 \times 0 x$

$0 \times 0 \times O X O X$

$\mathrm{m} \mathrm{m} \mathrm{m} \mathrm{m} \mathrm{m} \mathrm{m} \mathrm{-} \mathrm{alternation}$

In Example a there are only two matching elements, which is less than the length of the terminal run of the sequence (Length 3 ) and less than the number of matching elements in the previous line (four). Therefore, the matches on this line are deleted (indicated by placing them in brackets). The duplicate is then shifted left by one element, the procedure continued, and so on until the left end of the sequence is reached by the right end of the duplicate.

6. After the matching process is completed, the number of recorded subsequences preceding a run are cumulated and divided by the total number of recorded matching subsequences to provide the numeric value of the syntely of the pattern. The entire algorithm may easily be computerized.

\section{Some Complete Examples}

The stepwise matching shifts of the algorithm produce the following results in some real examples, where each line indicates a stepwise shift of the terminal segment one element to the left, the $\mathrm{ms}$ indicate subsequences matching the terminal segment pattern, and run and alt to the right of a dash indicate that a run or an alternation, respectively, was a consequent of the matched subsequences:

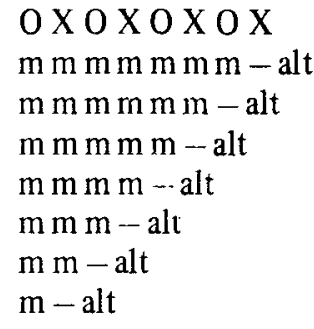

$0 \times \times \times 00 \times 0$

Syntely $=0 / 28=.0$

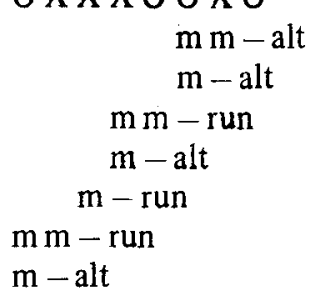

Syntely $=5 / 10=.5$

The previous examples illustrated the operation of the basic algorithm. Next consider the following sequences. These patterns illustrate the gestalt unitization of the terminal run. Since the terminal run is to be considered as a unit, it has no matching subsequences within itself. Similarly, in these examples, since the match of the terminal run is immediately available, the gestalt unit remains intact in its matching operations and is not subdivided. The stepwise matching shifts of the algorithm produce the following results:

$$
\begin{array}{ll}
\begin{array}{l}
X \times X \\
\mathrm{mmm}-\text { alt }
\end{array} & \begin{array}{c}
0 \times \mathrm{XXX} 000 \\
\mathrm{mmm}-\text { alt } \\
\mathrm{mmm}-\mathrm{run}
\end{array} \\
\text { Syntely }=0 / 4=.0 & \text { Syntely }=4 / 7=.57
\end{array}
$$

Finally, consider a pattern in which the unity of the terminal run must be broken because matches with subsequences of equal run length are not available (XXOXXOOO). On the first shift, a match with Run Length 2 is accepted. In general, since the algorithm proceeds from right to left in its matching operations, matches are accepted shorter than the terminal run only if the match is equal or greater than any match found to the right. With this example, the stepwise matching operation of the syntely algorithm produces the following result:

$$
\begin{gathered}
\begin{array}{c}
X X O X X O O O \\
m m-\text { alt }
\end{array} \\
m m-\text { alt } \\
\text { Syntely }=0 / 4=.0
\end{gathered}
$$

Using this straightforward algorithm, the syntely of any binary pattern, no matter what its length, may be found. A simple experiment was conducted to determine whether subjects' expectancies are described by this algorithm.

\section{EXPERIMENT I}

\section{Method}

Since the syntely algorithm as proposed so far does not yet take asymmetric event frequencies into account, it was decided to use sequences of equally frequent alternatives to test the adequacy of the present conception. Since the syntely algorithm 
Table 1

Subjects' Judgments and Stimulus Measures for Complexity, Symmetry, and Syntely

\begin{tabular}{|c|c|c|c|c|c|c|}
\hline \multirow{2}{*}{$\begin{array}{l}35 \text { Patterns } \\
\text { of } 8 \text { Binary } \\
\text { Elements }\end{array}$} & \multicolumn{2}{|c|}{ Complexity } & \multicolumn{2}{|c|}{ Symmetry } & \multicolumn{2}{|c|}{ Syntely } \\
\hline & J & $\mathrm{H}_{\text {code }}$ & $\mathrm{J}$ & .11 & $\begin{array}{c}\mathrm{J} \\
( \pm .5)\end{array}$ & $\begin{array}{c}.11 \\
( \pm .5)\end{array}$ \\
\hline XOXOXOXO* & 121 & 6.00 & 695 & .38 & .50 & .50 \\
\hline OXXOXOXO & 525 & 18.27 & 426 & .25 & .19 & .37 \\
\hline XXOOXOXO* & 535 & 18.27 & 329 & .22 & .19 & .29 \\
\hline OXOXXOXO* & 350 & 19.69 & 738 & .38 & .12 & .25 \\
\hline XOOXXOXO & 535 & 18.27 & 408 & .25 & .23 & .27 \\
\hline OOXXXOXO & 595 & 16.00 & 310 & .25 & .00 & .14 \\
\hline XXOXOOXO & 520 & 19.69 & 364 & .22 & .25 & .21 \\
\hline XOXXOOXO* & 315 & 18.27 & 425 & .19 & .06 & .08 \\
\hline OXXXOOXO* & 550 & 17.00 & 351 & .22 & .06 & .00 \\
\hline XXXOOOXO* & 365 & 16.00 & 281 & .28 & .31 & .05 \\
\hline OXOXOXXO* & 400 & 18.27 & 429 & .25 & .12 & .30 \\
\hline XOOXOXXO* & 575 & 19.69 & 389 & .19 & .25 & .17 \\
\hline OOXXOXXO & 505 & 17.00 & 390 & .25 & .31 & .04 \\
\hline XOXOOXXO & 520 & 18.27 & 365 & .25 & .06 & .04 \\
\hline OXXOOXXO* & 405 & 7.00 & 720 & .38 & .25 & .25 \\
\hline XXOOOXXO* & 430 & 17.00 & 409 & .28 & .31 & .23 \\
\hline OOXOXXXO* & 545 & 16.00 & 225 & .22 & .19 & .14 \\
\hline OXOOXXXO & 575 & 17.00 & 320 & .22 & .31 & .05 \\
\hline XOOOXXXO & 350 & 16.00 & 385 & .25 & .13 & .25 \\
\hline OOOXXXXO* & 300 & 6.81 & 376 & .34 & .31 & .30 \\
\hline XXOXOXOO & 540 & 18.27 & 379 & .25 & .50 & .50 \\
\hline XOXXOXOO & 555 & 19.69 & 336 & .22 & .44 & .50 \\
\hline OXXXOXOO & 575 & 16.00 & 231 & .22 & .06 & .07 \\
\hline XXXOOXOO* & 430 & 17.00 & 274 & .31 & .19 & .25 \\
\hline XOXOXXOO* & 480 & 18.27 & 350 & .22 & .50 & .50 \\
\hline OXXOXXOO* & 510 & 17.00 & 420 & .25 & .44 & .50 \\
\hline XXOOXXOO & 250 & 7.00 & 595 & .25 & .50 & .50 \\
\hline OXOXXXOO* & 535 & 16.00 & 285 & .25 & .19 & .10 \\
\hline XOOXXXOO & 545 & 17.00 & 343 & .28 & .19 & .06 \\
\hline OOXXXXOO & 255 & 6.81 & 730 & .44 & .06 & .10 \\
\hline XXXOXOOO & 420 & 16.00 & 380 & .25 & .44 & .50 \\
\hline XXOXXOOO* & 540 & 17.00 & 279 & .31 & .37 & .50 \\
\hline XOXXXOOO* & 395 & 16.00 & 300 & .28 & .50 & .50 \\
\hline OXXXXOOO* & 320 & 6.81 & 380 & .34 & .13 & .07 \\
\hline XXXXOOOO & 147 & 6.81 & 740 & .38 & .44 & .50 \\
\hline
\end{tabular}

Vote- $J=$ judged, $M=$ measured

*The first seven elements from the left were used as stimuli in Experiment $I I$.

is designed to measure all of the intrinsic structure available in a pattern that influences expectancy, it was decided to present the patterns in such a way as to reduce as much as possible any disturbing influences of faulty memory. Therefore, each pattern was presented all at once rather than sequentially. All 70 possible sequences of four Xs and four Os were used. These were segregated in to two sets of 35 sequences, so that in either set all sequences were different and each had a complementary sequence in the other set. For each of these sets of 35 sequences, 35 different orders of the sequences were printed in a vertical column on computer sheets so that each sequence appeared once at each position of the vertical column. An ordered set of these sequences is shown in Table 1.

These 70 lists were presented to 70 subjects of an introductory psychology class at Southern Connecticut State College. The subjects were not informed about the purpose of the experiment. They were told simply to read each sequence of eight elements from left to right and, for each of the 35 sequences on their page, to write the element (either an X or an O) that they thought should come next in the sequence if the sequence was continued. They were then told not to worry or spend a lot of time on the sequences, but just to read them and put down whatever they felt should happen next.

\section{Results}

The proportion of responses that were runs (continuations of the terminal run) were tabulated for each sequence, and the two complementary sets were compared. The correlation coefficient for the proportions of run responses between the two complementary sets of 35 sequences was $.89, p<.001$. indicating a reliable effect relatively uncontaminated by the nature of the elements of the sequences, but dependent instead on the patterns of the sequences. These proportions were averaged for the complementary pairs and correlated with the measured syntely of the sequences, yielding a coefficient of $.86, p<.001$. The scattergram for this comparison is shown in Figure 1. Each point is based on an $\mathrm{N}$ of 70 .

It should be clear from the scattergram and the correlation coefficient that the syntely algorithm provides an excellent fit to the expectancy predictions of the subjects in this experiment. The fit is this good in spite of the fact that the algorithm is parameter free, and no minor adjustments can be made to accommodate the data. No other models based on intrinsic structure exist that make explicit predictions for comparison with these results other than a probability matching model, which would predict a run proportion response of .5 for each sequence, and Restle's (1961) model, which is inapplicable to 11 of the 35 patterns which have no run lengths equal or longer than the terminal run and predicts run proportions of $.0, .67$, or 1.0 for the rest of the sequences. Nithough it is inappropriate for almost one-third of the sequences, Restle's model does take into account a portion of the intrinsic structure of the patterns, namely, run lengths, and so it does have some

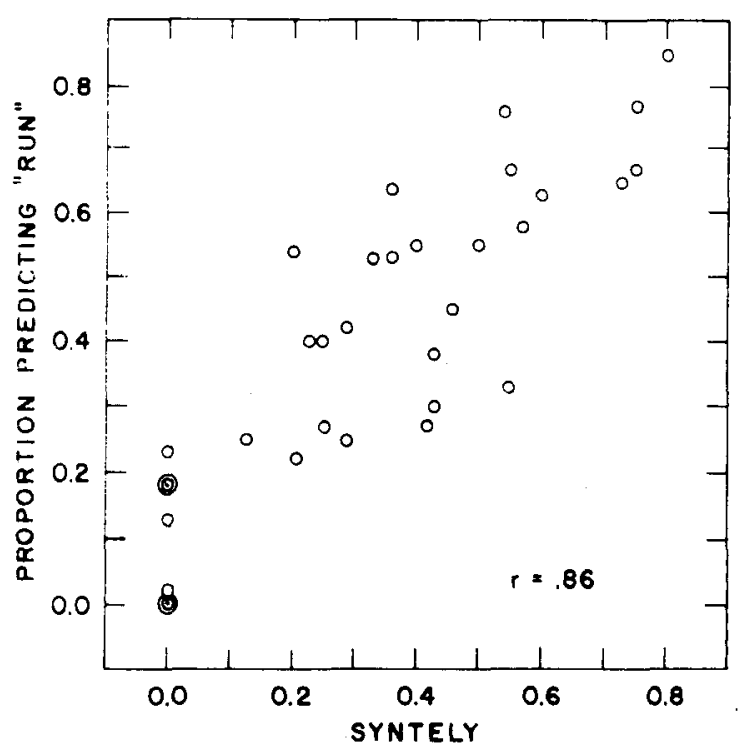

Figure 1. The proportion of subjects predicting a "run" for each of the 35 sequences of eight binary elements as a function of the syntely of those sequences, where syntely is a stimulus measure of pattern continuation. 
predictive power. For the 24 sequences where it is appropriate, the correlation coefficient of Restle's model with subjects' predictions is .54 , whereas the syntely correlation with subjects' results is 81 . Somehow subjects are responding to the intrinsic structure of the sequences by matching their predictions with the frequency of the alternative consequences as provided in a syntely analysis based on the inductive principle.

A plausible process by which a subject chooses a prediction from the set of alternatives in the sequence pattern is given by stimulus sampling theory: The matched subsequence consequents are available in a pool for each subject from which they are sampled. The predictions of a large group of subjects thus reflects the availability of the alternatives.

However, it is just as plausible to suppose that at some level, somehow, subjects are individually calculating the syntely of each pattern and making their response on the basis of this calculation and some idiosyncratic decision rule. If this were the case, extensive observations on a single subject might eventually shed light on the nature of this process.

But within the present frame of our ignorance, it is unnecessary to take either of these explanatory steps. It is sufficient to show that a functional relationship exists between the intrinsic structure of the patterns as derived by the syntely analysis and the predictions of a group of untrained subjects, and that is what this experiment has done.

Other approaches not based on a direct measure of the stimulus structure, using instead the response patterns of subjects to the stimulus, are possible. One such method has been proposed by Lordahl (1968, 1970) to account for sequential predictions by the weighted sum of several hypotheses. A very good fit to the data may be achieved through the selection of hypotheses and weighting parameters. The parsimony of this approach depends inversely on the number of parameters needed. Lordahl found it necessary to use 40 different parameters, corresponding to particular hypotheses related to perceived pattern structures in the binary stimulus sequences.

\section{Simplicity and Expectancy}

A still different strategy for understanding expectancy with binary patterns might argue that subjects predict the next element that makes the resulting sequence in some sense simpler or less complex. Several models of the simplicity of binary patterns have been proposed. These models can be partitioned into two classes, those based on response measures and those based on stimulus measures (Vitz, 1968). Among response measures, the mean verbal length of the description of the sequences by human subjects has been used (Glanzer \& Clark, 1962), as has the response uncertainty of the preferred start point of the pattern (Royer \& Garner, 1966). These analyses will not be treated in detail since the primary concern of this paper lies with stimulus measures of pattern structure. A stimulus measure of pattern complexity has been proposed by Vitz (1968) based on an information measure of the run structure of the patterns, and this measure will be used to test the argument first.

None of the models has been applied to sequential prediction data, and no specific numeric assessment of subjects' predictions has been drawn from them. However, all three models correlate highly with each other and with the number of runs in a sequence. In fact, Vitz's measure $H_{\text {run-span }}$ is simply $\log _{2}$ of the total number of runs in a pattern. It follows that they should predict that, in order to make the resulting pattern as simple as possible, subjects should mainly continue the terminal run of a sequence. In fact, only for 14 of the 35 patterns tested did the majority of subjects continue the terminal run, a number not different from chance. This also eliminates the possibility that subjects were continuing the sequences in such a way as to make the resulting sequences as complex as possible in terms of the number of runs in the sequence.

A related model of the complexity of binary and trinary sequences based on stimulus measures has been offered by Vitz and Todd (1969). They have provided numeric values for the complexity of binary patterns of Length 8 . These are reproduced in Table 1 under the column headed $\mathrm{H}_{\text {code }}$. A detailed description of how to evaluate $\mathrm{H}_{\text {code }}$ can be found in the original paper. Unly a description of the principles involved will be given here. Essentially, Vitz and Todd constructed an information theoretic measure of pattern complexity based on the number and length of coded elements. These elements are found in a hierarchic series of encoding levels, and they are based on the detection in the sequences of runs of individual symbols and runs of coded subpatterns at each coding level. As a result, there is a very high correlation between their information measure and a simple count of runs (Simon, 1972). In fact, the correlation between $\mathrm{H}_{\text {code }}$ and the number of runs in a sequence varies from .79 to .92 for different sets of sequences (Simon, 1972, p. 377). Accordingly, a similar result may be expected for the $\mathrm{H}_{c \text { ode }}$ measure as was found for the run measure already tested. However, $\mathrm{H}_{\text {code }}$ correlates higher with direct measures of complexity than does the run length of the pattern for all sets of sequences, so $\mathrm{H}_{\text {code }}$ may be a better measure of complexity and produce different results. In order to test the possibility that the previous measure of complexity, the number of runs in the sequence, was inadequate and that subjects really were continuing the sequences with the element that made the resulting sequence in some sense simpler, another simple experiment was carried out. 


\section{EXPERIMENT II}

\begin{abstract}
Method
Nineteen different sequences of four Xs and three Os were selected randomly for one list, and their complementary set of 19 sequences of three Xs and four Os formed the other list. These 19 patterns are shown with asterisks in Table 1. For each list 16 random orders were created and printed, each in a vertical column of a computer output sheet. Each of 32 uninformed subjects of an introductory psychology class at Southern Connecticut State College was given one of these 32 lists and, with the same instructions as given in Experiment I, was asked to write on the sheet the element he thought should come next in the sequences.
\end{abstract}

\section{Results}

The correlation of the proportion of run responses between the two sets of 19 sequences, for a sequence and its complement, was $.84, \mathrm{p}<.001$, indicating again a reliable response to the pattern of each sequence. The correlation of the mean of these two sets of proportions with the syntely of each sequence as determined by the application of the algorithm was $.83, p<.001$. In spite of the inequality in the frequency of the alternative elements in a sequence $(4: 3)$, the syntely algorithm still provided a good fit to the data. Moreover, looking only at whether the proportion of subjects responding run was greater or less than .5 when the syntely measure was greater or less than .5 for each sequence, the sign was the same for 18 of the 19 patterns. In the remaining sequence the syntely measure had a value of .5 exactly.

Using these results, the $\mathrm{H}_{\text {code }}$ values provided by Vitz and Todd (1969) for binary patterns of Length 8 can be used to see if subjects are continuing each sequence with the element that makes the resulting sequence somehow simpler. If this is the case, then the majority of subjects should select the simpler of the two possible sequences as measured by $\mathrm{H}_{\text {code }}$. In fact, this was true for only 9 of the 19 sequences. This result makes it implausible as well that subjects select the element that makes the resulting sequence more complex.

These results raise the general question of whether there is any relation between the syntely measure or subjects' predictions and the simplicity of the binary patterns. It is clear that subjects do not select the next element in order to make the resulting sequence simpler or more complex. But perhaps there exists nevertheless a relation between the simplicity of the sequence and the degree of agreement among subjects about what should come next. Before trying to answer this question, the relation between syntely and symmetry will be established.

\section{Symmetry and Syntely}

In an analysis of the results of the famous Zenith radio experiments on telepathy, Goodfellow (1938) showed convincingly that the bilateral symmetry of the five-element-long binary patterns had a strong influence on the frequency of their choice by listeners of the radio program. The listeners shunned the symmetric sequences as possible answers to the telepathic message. Using a very similar analysis which they chose to call subsymmetry, Alexander and Carey (1968) showed that the cognitive simplicity of 35 binary patterns of Length 7 was almost perfectly accounted for by the relative numbers of subsymmetries in the different patterns.

The syntely measure proposed in this paper bears some striking similarities to this measure of symmetry. Syntely is in fact based technically on a kind of symmetry, translational symmetry. The finding of matches to the terminal segment by moving that segment laterally detines lateral subsymmetries. Symmetry in the more common sense of the word is bilateral symmetry, defined about the median axis of the sequence. This is the sense of symmetry used in this paper.

A simple algorithm provides a numeric measure of the symmetry of binary patterns that is very similar to Goodfellow's measure and correlates perfectly with Alexander and Carey's subsymmetry. The similarity with the syntely algorithm lies basically in the use of an autocorrelational technique to find matching subsequences; but the matching for symmetry is done about the median axis instead of from the rightmost end, the pattern is matched against its reflected self rather than against a duplicate sequence, and the matching is performed on the elements themselves rather than on the pattern of runs and alternations, as in the syntely algorithm. Autocorrelation mechanisms have been proposed by a number of theorists as ideal ways of describing certain processes in perception (Dodwell, 1971) and in memory (Anderson, 1973). The symmetry and syntely algorithms proposed in this paper extend the use of autocorrelation to a new area of perception and to the grand domain of future expectancy.

\section{Symmetry Algorithm}

The steps for carrying out the symmetry algorithm begin with taking the binary pattern and aligning its reflected self below it as in the following example:

\section{$\mathrm{XXO} \times \mathrm{XOXO}$ OXOXOOXX}

Compare the reflection of this pattern vertically, indicating a match with an $\mathrm{m}$ and a mismatch with a dash. Matching here is defined by a physical match of the elements, not a match of the runs and alternations, as in the syntely algorithm. Continue the comparison by shifting the top pattern one-half space to the left and the reflected pattern one-half space to the right. By shifting each one-half space the results are aligned vertically: 


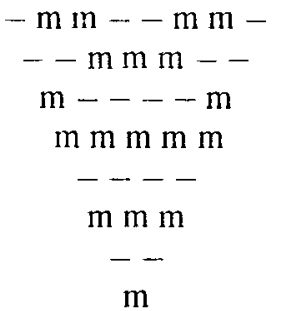

And then begin again and reverse the shifts of each pattern:

$$
\begin{gathered}
-\mathrm{mm}-\mathrm{mm}- \\
\mathrm{m}--\mathrm{m}-\mathrm{m} \\
-\mathrm{m} \mathrm{mm}- \\
\mathrm{m}-\mathrm{m}-\mathrm{m} \\
---- \\
-\mathrm{m}- \\
\mathrm{mm}
\end{gathered}
$$

$\mathrm{m}$

Since the comparisons are completely symmetrical, they can be reflected on themselves and the median axis removed:

$$
\begin{array}{r}
-\mathrm{m} \mathrm{m-} \\
--\underline{\mathrm{m}} \\
\mathrm{m}-- \\
\mathrm{mm} \\
-- \\
\underline{\mathrm{m}} \\
- \\
\mathrm{m} \mathrm{m-} \\
\mathrm{m}-- \\
-\mathrm{m} \mathrm{m} \\
\hline \mathrm{m}- \\
-- \\
-
\end{array}
$$

Finally, the matched, and thus symmetric, elements are counted on each line in from the right until the first dash is encountered, at which point the algorithm shifts to the next line. The sum of these matches (the underlined $\mathrm{ms}$ in the above example) is divided by the total number of comparisons for the pattern, and the quotient is then the numeric measure of the symmetry of the pattern. In this example, the number of symmetric elements is 7 (the underlined $\mathrm{ms}$ ) and the

\begin{tabular}{|c|c|c|c|c|c|}
\hline & \multirow{2}{*}{$\begin{array}{c}\mathrm{H}_{\text {code }} \\
\text { Com- } \\
\text { plexity }\end{array}$} & \multicolumn{2}{|c|}{ Symmetry } & \multicolumn{2}{|c|}{ Syntely } \\
\hline & & $\mathbf{J}$ & $\mathrm{M}$ & $\begin{array}{c}\mathrm{J} \\
( \pm .5)\end{array}$ & $\underset{( \pm .5)}{\mathrm{M}}$ \\
\hline Judged Complexity & .74 & -.70 & -.68 & -.30 & -.31 \\
\hline $\mathrm{H}_{\text {code }}$ Complexity & & -.58 & -.71 & -.17 & -.12 \\
\hline Judged Symmetry & & & .71 & .12 & .29 \\
\hline Measured Symmetry & & & & .06 & .11 \\
\hline Judged Syntely $( \pm .5)$ & & & & & .77 \\
\hline
\end{tabular}
total number of comparisons is 32 . The symmetry of this pattern is then $7 / 32=.22$. This algorithm may easily be extended to any binary pattern of any length to determine its bilateral symmetry, as a proportion ranging from 0 to 1 .

Given this measure of the intrinsic structure of binary patterns, how does it relate to the judged complexity of
Table 2

Correlation Coefficients Among Subjects' Judgments and Stimulus Measures of Complexity, Symmetry, and Syntely: $\left.\mathbf{r}_{(p}<.05\right)=.30$

Note-J = judged, $M=$ measured

the sequences? Is there a stronger relation with the judged symmetry of these patterns? Does it bear any relation to the syntely of the patterns? If there does exist a relation between symmetry and syntely, it may be that, like the relation between simplicity and syntely, it is not straightforward.

\section{EXPERIMENT III}

\section{Method}

Twenty-four Yale undergraduates fulfilling an introductory psychology course requirement were tested individually as members of three experimental groups of eight. They were each presented with 70 cards $2 \frac{1}{2} \times 3 \frac{1}{2}$ in. in size, each of which had one of the 70 possible patterns of four Xs and four Os centered on it, and were asked to go through the cards one at a time, each group doing only one of the three following things: (1) Read the pattern aloud from left to right and judge its complexity by giving a number from 1 to 50 that reflected how complex the pattern seemed, the higher the number the greater the complexity. (2) Read the pattern aloud from left to right and judge its symmetry by giving a number from 1 to 50 that reflected the symmetry of the pattern, the greater the symmetry the greater the number. (3) Read the pattern aloud from left to right and then say the element, either an $X$ or an $O$, they felt should come next if the sequence continued (judge syntely).

\section{Results}

The responses of all three groups were first sorted arbitrarily into two complementary sets of 35 sequences so that in each set all the sequences were different and each had a complementary sequence in the other set. Then, for each sequence, the eight complexity judgments were summed, the eight symmetry judgments were summed, and the proportion of "run" responses was subtracted from .5 and given an absolute value. The correlations between the two complementary sets of 35 sequences for the judged complexity, symmetry, and the deviation of the syntely judgments from .5 were .88 , 96 , and .79 , respectively, indicating again a high reliability of the subjects' responses to the structure of the patterns.

These response measures were then collapsed across complementary sequences and are presented in Table 1 , along with three stimulus measures of complexity, symmetry, and syntely. What is of interest are the interrelations among this set of response and stimulus 
measures. In Table 2 are shown the correlations among this set.

The most striking result is that the significant correlations split simply into two groups, either dealing with pattern simplicity or else with pattern continuation. The measures in each group correlate highly with each other but not with the measures in the other group. It seems that pattern simplicity or symmetry is not related to the strength of judged pattern expectancy or syntely.

The syntely measure correlates well only with the judged syntely, when both are taken as deviations from .5 . The symmetry measure correlates well with both judged complexity and judged symmetry as well as $\mathrm{H}_{\text {code }}$, all measures of pattern simplicity. The only further interest lies in the slight but still significant correlations of the judged syntely and the syntely measure with judged complexity, but not with the other measures. This represents a relatively slight dissociation between the relative contributions of sequential regularity and symmetry to the simplicity of patterns, insofar as syntely measures sequential regularity and not symmetry.

It is clear that both $\mathrm{H}_{\text {code }}$ and the symmetry measure evaluate some psychologically relevant aspects of the patterns for their simplicity. Whether they evaluate the same, different, or overlapping aspects of the patterns is not clear. The high positive correlation between $\mathrm{H}_{\text {code }}$ and the symmetry measure gives reason to believe that they measure the same aspect, but it may be useful in the future to search for patterns that prove to be simple but not symmetric and vice versa, in order to determine whether $\mathrm{H}_{\text {code }}$ and the symmetry measure are sensitive to different aspects of the patterns. There is little evidence in the present data that these patterns can be found. However, it would seem likely that for much longer sequences the correlation between symmetry and simplicity should break down, while $\mathrm{H}_{\text {code }}$ may still be useful.

\section{Errors in the Tracking of Recurrent Binary Sequences}

The syntely algorithm has another application that may be used to test its descriptive power. Consider the situation in which a subject predicts each next element of an unknown binary sequence as it is presented recurrently one element at a time. The subject will err in his predictions at those points where his expectancy does not match the pattern, and those errors will continue until the subject remembers the pattern fully. The errors at each point may be accumulated and normalized (divided by the total number of errors for the whole pattern) to provide an error profile for each pattern. The number of elements presented up to the last error constitutes the number of trials to criterion.

A similar profile may be derived from the syntely algorithm in a straightforward way by measuring the

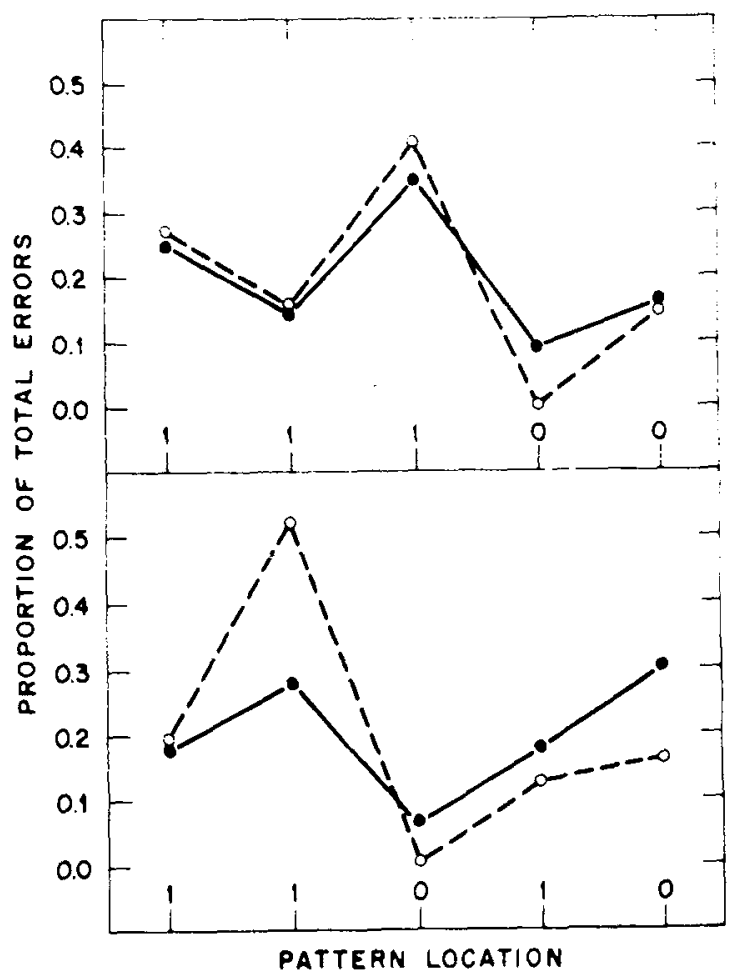

Figure 2. Ermor profiles for a simple and a complex pattern. The filled circles indicate the proportion of the total errors for the pattern at each location, from Garner and Gottwald (1967). The open circles indicate the proportion given by syntely analysis normalized for the pattern.

syntely at each point of a repetitive string of the basic sequence. The empirical result of such an application of the syntely algorithm is that there exists a point somewhere in the string after which the syntely measure is greater than .5 when there is a run in the string, and less than .5 when there is an alternation in the string. The syntely algorithm may be said to be correctly predicting the string from that point on. The number of elements needed in the string to reach this point can be taken as a simple estimate of the number of trials to criterion. The absolute deviation of the syntely measure from .5 may be taken as an estimate of the likelihood of an error at this point. If these absolute deviations are normalized for the first full cycle of the sequence that is correctly predicted by the syntely algorithm, at each location of the pattern, the resulting profile may be compared directly with the subjects' actual error profiles. In this way, the syntely algorithm may be used to predict the relative number of errors at each location and the estimated trials to criterion for any pattern without any conversion parameters.

The first application of the syntely algorithm is to the error data provided by Garner and Gottwald (1967). They asked their subjects to predict each element of a recurrent binary sequence as it was presented visually at the rate of one element every $4 \mathrm{sec}$. They used only two sequences, an easy one $(X \times X O O)$ and a difficult 


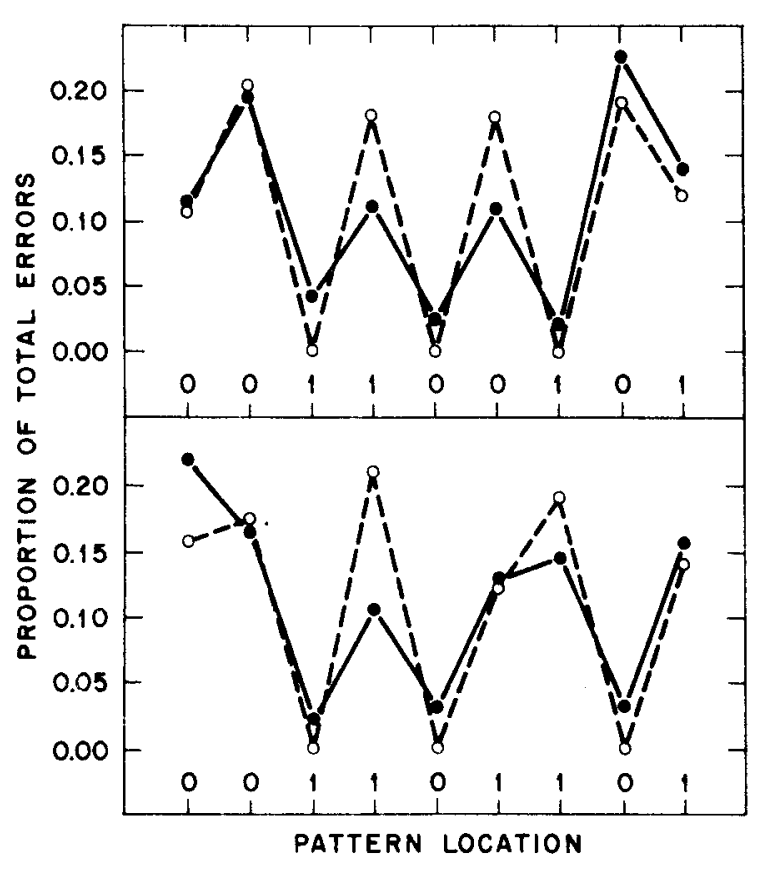

Figure 3. Error profiles for two binary patterns. The filled circles indicate the proportion of the total errors for each pattern at each location found by Restle (1967). The open circles indicate the normalized proportion at each location given by a syntely analysis.

sequence (X X O X O) and began each pattern at each of the five different starting points for different subjects. The actual locations of errors were recorded for each pattern. The mean proportion of the total errors for each pattern, pooled over all starting points, are shown at each location in Figure 2. The normalized error profile as given by the syntely algorithm is shown in Figure 2 for comparison. The fit of the two profiles is excellent for the simple pattern, and the error transitions are followed faithfully in the complex pattern. Garner and Gottwald recorded a number of other response measures as well, among them the mean location in the sequence of the element or trial following the last error. These mean numbers of trials to criterion were 16.3 for the simple pattern and 25.8 for the complex pattern. The corresponding lengths of string used by the syntely algorithm before perfect prediction were 17 and 25 , respectively.

Another set of error profiles is provided by Restle (1967). Subjects again predicted recurrent cycles of two unknown sequences, a hard one (O OXXOXXOX) and an easy one $(0 \circ X X O O X O X)$. The sequences were presented visually at the rate of about one element every $2 \mathrm{sec}$. The actual normalized error profiles along with the normalized profiles given by the syntely algorithm are shown in Figure 3. Again a very good fit to the profile transition is apparent. The mean location of the trial following the last error is not provided for the real subjects, but the length of string needed for the syntely algorithm was 26 elements for the easy pattern and 28 elements for the hard pattern.

\section{CONCLUSION}

The accumulated success of the syntely measure in predicting the subjects' responses in a sequential prediction task and their error profiles when they predict recurrent sequences, its relation to a proven measure of the symmetry and thus the simplicity of short binary sequences, and its potential usefulness for dissociating separate sequential and symmetric aspects of the intrinsic structure of binary patterns provides good evidence that the syntely algorithm, and the inductive principle upon which it is based, measures psychologically relevant aspects of intrinsic stimulus structure.

It must be emphasized that the algorithm, intended to measure the syntely of binary sequences, is, as it is presented in this paper, only a first step, a first approximation to the practical application of the principle of induction to the problems of understanding human expectancy. The key to understanding human expectancy, at least with binary patterns, lies in measuring the intrinsic structure of the patterns. The principle of induction provides the essential rule for producing this measure: match the present events to the past and weigh the past consequences of those events that match. This matching operation effectively defines the process of autocorrelation, a process which has been proposed by many as a basic biologic activity either directly (Kabrisky, 1964) or indirectly as part of a holographic system (van Heerden, 1968). It is very satisfying indeed to extend the applicability of this potentially powerful analogy from the two main psychological domains where its usefulness has been demonstrated, the past and the present, memory and perception, to the third major domain of psychology, the future, expectancy. The process of autocorrelation is, however, subservient to the general principle of induction and may turn out not to be the best means of implementing this principle in the long run.

As it has been appleed in its present form, the principle of induction has been shown to be an altogether adequate basis for describing the inferences subjects make after reading short binary sequences, even when the intrinsic structure of those sequences has not been constrained in any artificial or extrinsic way. This is an important consideration and represents a step toward greater generality beyond the current inferential models outlined and summarized so beautifully by Simon (1972), which depend on the existence of regularities within the binary sequence based on rules. The success of the syntely algorithm shows that subjects can be responsive to the regularities of a pattern without resorting to the formulation of rules, but simply as the 
result of a basic inferential process that depends on memory, as long as the regularities are expressed in the intrinsic structure of the stimulus.

\section{REFERENCES}

Alexander, C., \& Carey, S. Subsymmetries. Perception \& Psychophy sics, 1968, 4, 73-77.

Anderson, J. A. A theory for the recognition of items from short memorized lists. Psychological Review. 1973, 80,417-438.

Anderson. N. H. Effect of first-order conditional probability in a two choice leaming situation. Journal of Experimental Psychology, 1960, 59, 73.93

Butler, P. A., Myers, N. A.. \& Myers. J. L. Contingencies among event runs in binary prediction. Journal of Experimental Psychology, 1969, 79, 424-429.

Dodwell, P. C. On perceptual clarity. Psychological Review $1971,78,275-289$.

Feldman, J., \& Hanna, J. F. The structure of responses to a sequence of binary events. Joumal of Mathematical Psy chology, 1966, 3, 371-387.

Galanter, E. H., \& Smith, W. A. Some experiments on a simple thought problem. American Journal of Psychology, 1958,71. 359-366.

Gambino, B.. \& Myers, J. L. The role of event runs in probability learning. Psychological Review, 1967, 74, 410-119.

Garner, W. $\mathbf{R}$. The processing of information and structure. Potomac: Erlbaum, 1974

Garner, W. R., \& Gottwald, R. L. Some perceptual factors in the learning of sequential patterns of binary events. Journal of Verbal Leaming and Verbal Behavior, 1967, 6, 582-589.

Gamer, W. R., \& Gottwald, R. L. The perception and learning of temporal pattems. Quarterly Joumal of Experimental Psychology, 1968, 20, 97-109.

Glanzer, M., \& Clark, W. H. Accuracy of perceptual recall: An analysis of organization. Journal of Verbal Leaming and Verbal Behavior, 1962, 1, 289-299.

Goodfellow, L. D. A psychological interpretation of the results of the Zenith radio experiments in telepathy. Journal of Experimental Psy chology, 1938, 23, 197-200.

Goodnow, J. J., \& Pettigrew, T. F. Effect of prior patterns of experience upon strategies and learning sets. Journal of Experimental Psy chology, 1955, 49, 381-398.

Hake, H. W., \& Hyman, R. Perception of the statistical structure of a random series of binary sy mbols. Journal of Experimental Psychology, 1953,45, 64-74.

Handel, S., \& Buffardi, L. Pattern perception: Integrating information presented in two modalities. Science, 1968, 162, $1026-1028$.
Jenkins. W, O., \& Stanies, I. C.. Jr. Partial reinforcement: A review and a critique. Pyychological Bulletin, 1950, 47. $153 \div 234$.

Jones, M. R. From probubility learning to sequential processing: A critical review. Psychological Bulletin, $1971,76,153-185$.

kabrisky, If. A proposel model for visual information procesimg in the human broin. Urbana: University of Illinois Press, 1964

Lordahi, D. S. Pattern perception and the use of hypotheses in prediction of binary events. Proceedings of the 76th Annual Convention of APA $1968, \$ 8-50$

Lordahl. D. S. An hypothesis approach to sequential predictions of binary events. Joumal of Mathematical Psychology, 1970. 7. $3339-361$.

vies, $R$ C Effects of probable outcome information on two-choice learning. Journal of Experimental Psychology. $1962,64.430-433$.

Preusser, D., Ganter, W. R.. \& Gottwald, R. L. Perceptual oranization of two-lement temporal patterns as a function of their component oneelement patterns. American Journal of Psychology, 1970,83, 151-170.

Reichenbach. H. The rise of scientific philosophy. Berkeley: University of California Press, 1968.

Restle, F. The psy chology of judgment and choice. New York: Wiley, 1961 .

Restle, F. Run structure and probability learning: Disproof of Restle's model. Journal of Experimental Psychology, 1966 $72,383-389$

Restle, F. Grammatical analysis of the prediction of binary events. Joumal of Verbal Leaming and Verbal Behavior, 1967. $6,17-25$.

Royer, F. L., \& Gamer, W. R. Response uncertainty and perceptual difficulty of auditory temporal patterns. Perception \& Psy chophysics, 1966, 1, 41-47.

Schoonard. J. W.. \& Restle, F. Analy sis of double alternation in terms of patterns of stimuli and responses. Journal of Experimental Psychology, 1961, 61, 365-367.

Simon, H. A. Complexity and the representation of patterned sequences of symbols. Psychological Review, 1972, 79 369-382,

van Heerden, P. J. The foundation of empirical knowledge. Wassenaar: N. V. Uitgeverij Wistik, 1968.

$V i t z, P$. C. Information, run structure, and binary patterm complexity. Perception \& Psychophysics, 1968, 3, 275-280.

Vitz, $P$, \& Todd, $R$. C. A coded element model of the perceptual processing of sequential stimuli. Psychological Review, $1969,76,433-449$.

(Received for publication September 5, 1974; revision accepted October $27,1974$. 